\title{
Processing by Local Interneurons of Mechanosensory Signals Involved in a Leg Reflex of the Locust
}

\author{
M. Burrows* and H-J. Pflüger ${ }^{*}$ \\ "Department of Zoology, University of Cambridge, Cambridge CB2 3EJ, England, and ${ }^{*}$ Fachbereich Biologie, \\ Universität Konstanz, D7750 Konstanz, Federal Republic of Germany
}

\begin{abstract}
At the distal end of the tibia of a locust hind leg are 2 pairs of movable spurs that can be moved by contact with external objects - as, for example, when the body sways from side to side and loads one leg unevenly or when the foot is placed on rough ground - but not by direct muscular action. Movements imposed on a spur evoke phasic bursts of spikes in the axon of a single receptor cell at its base. If the displacement is maintained, however, the response adapts within a few seconds.

The afferents from these spur receptors excite particular spiking local interneurons with cell bodies at the ventral midline of the metathoracic ganglion. Each afferent spike is consistently followed at a constant latency by a depolarizing potential in one of these interneurons. The potential can evoke a spike, and its amplitude is enhanced by a hyperpolarization applied to the interneuron. The central delay to this chemically mediated EPSP, which also includes conduction time to synaptic sites, probably indicates a direct connection.

Some spiking local interneurons are excited by the 2 anterior spurs but are unaffected by the 2 posterior ones, while others receive the converse pattern of inputs. The receptive fields of these interneurons also include regions on either the anterior or posterior surfaces of the tibia with excitatory inputs from hair afferents.

A reliable inhibitory reflex effect on the single levator tarsi is evoked by movement of any of the 4 spurs. The inhibitory potentials are not caused directly by the sensory afferents but involve the spiking local interneurons upon which the afferents synapse. The receptive field of this motor neuron therefore results from the convergence of inputs from a few interneurons. Motor neurons of other tarsal muscles are unaffected by movement of the spurs, but those of some more proximal muscles may be excited. These reflex effects should enhance the traction of the tarsus with the ground.
\end{abstract}

When a walking animal places its foot on the ground, its CNS will receive a barrage of sensory signals from the receptors that are inevitably activated. During normal walking, a pattern of sensory signals will be generated that indicates contact with the ground, load bearing during the stance phase, and the release of load that will be a signal leading to the start of the next swing phase. If the balance of the step is inappropriate or the footing insecure, then a corrective change in the motor output must be made during that step. This requires that there be quick reflex pathways able to respond to an unusual afferent signal and produce local adjustments of the motor output. For example, a cat

\footnotetext{
Received Jan. 2, 1986; accepted Feb. 14, 1986.

This collaboration was made possible by a Twinning Grant from the EEC commission. M.B. is supported by NIH Grant NS 16058 and H-J.P. by Grant PF1 28/3-2 from the D. F. G. We thank B. L. Watkins for the scanning electron microscopy and $G$. Laurent for the drawings.

Correspondence should be addressed to Dr. M. Burrows at the above address. Copyright $@ 1986$ Society for Neuroscience $0270-6474 / 86 / 092764-14 \$ 02.00 / 0$
}

bumping its paw against an external object will make appropriate adjustments to the step that is in progress (Forssberg, 1979). Touching the dorsal surface of the foot of a stick insect while it is standing causes the leg to be raised (Walther in Bässler, 1983), and imposed movements of the tibia during walking evoke a reflex with negative feedback that opposes this movement (Cruse and Pflüger, 1981).

On the hindleg of a locust are 2 pairs of movable spurs near the tarsus that are ideally placed to initiate local compensatory adjustments of the motor output to the leg muscles. They will be moved only if the body weight is transferred unevenly to one side or if the tarsus is placed on rough terrain. They are not normally deflected when the locust walks on a flat horizontal surface, and they cannot be moved by contraction of the tarsal muscles. Here we investigate the processing of the signals provided by receptors in these spurs.

In the segmentally arranged ganglia of insects, a group of local interneurons receives inputs from specific arrays of mechanoreceptors on the surface of a leg (Burrows and Watkins, 1986; Siegler and Burrows, 1983, 1984). The receptive field of an interneuron that is formed in this way can be large, encompassing much of a leg, or small and restricted to a region around a joint. The longitudinal boundaries of the fields correspond to the anteroposterior or dorsoventral axes of the leg, so that, for example, hairs on the dorsal surface of the femur will excite one interneuron, while hairs on the anterior surface of the tibia will excite another (Burrows and Siegler, 1985). The surface of the leg is thereby mapped onto these interneurons, which thus provide the first assessment of this modality of afferent signal. The organization of the complex receptive fields can be explained most easily by the role that these interneurons play in the many local reflexes that occur if specific receptors on a leg are stimulated (Siegler and Burrows, 1986). Some of the interneurons connect directly with particular leg motor neurons, so that they form the central element in pathways that mediate these local postural reflexes (Burrows and Siegler, 1982). All this evidence points to a crucial role for these interneurons in the adjustment of leg movements as a result of signals from external mechanoreceptors. We show here that a small number of these spiking local interncurons reccive direct inputs from a single receptor cell at the base of each of the movable spurs at the joint between the tibia and tarsus of a locust hindleg. Whenever a spur is moved, the spikes in an afferent excite these local interneurons and inhibit the single levator tarsi motor neuron in a consistent reflex.

\section{Materials and Methods}

Adult male and female locusts, Schistocerca gregaria (Forskal), were taken from crowded cultures in Konstanz or Cambridge. A locust was mounted ventral surface uppermost with the tibia of the left hindleg fully extended and held firmly in Plasticine. The tarsus of this leg and the tibiae and tarsi of all other legs, as well as the head and abdomen, were free to move. The meso- and metathoracic ganglia were exposed 

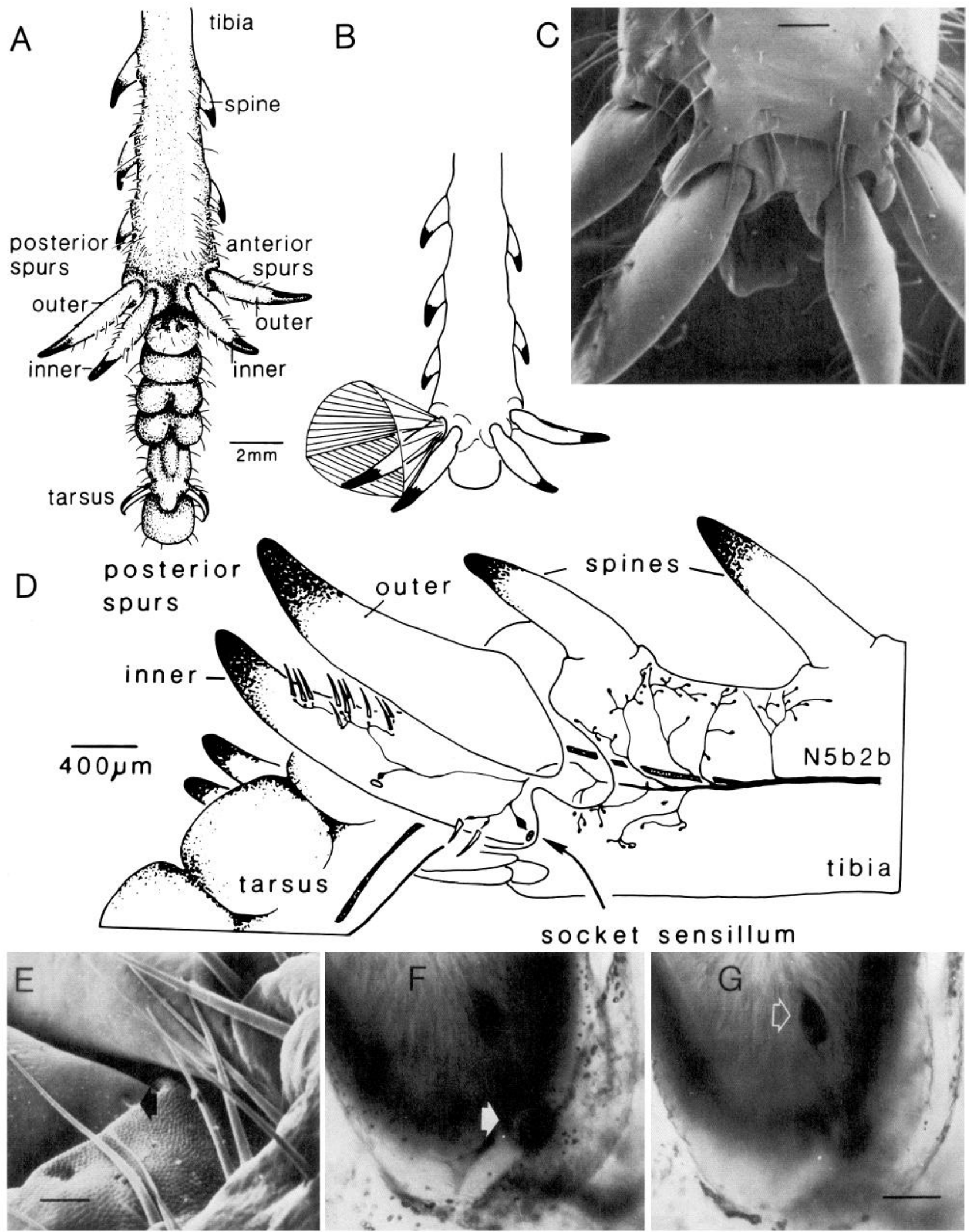

Figure 1. Morphology of the spurs and their receptors. A, Drawing of the tarsus and distal end of the tibia viewed ventrally. B, Diagram showing the extent of movement of a spur, illustrated for the outer posterior spur. $C$, Scanning electron micrograph of the insertion of the spurs at the distal end of the ventral tibia. $D$, Drawing of cobalt-stained posterior tibial nerve showing some of the receptors it innervates in the inner posterior spur and in the distal tibia. $E$. Scanning electron micrograph of the base of a spur showing the large campaniform (socket) sensillum (arrow). $F$ and $G$, Light micrographs at different focal planes of the socket sensillum (solid arrow) and its associated receptor cell body (open arrow) stained with cobalt. Calibration bars: $C, 200 \mu \mathrm{m} ; E, 50 \mu \mathrm{m} ; F$ and $G, 40 \mu \mathrm{m}$. 

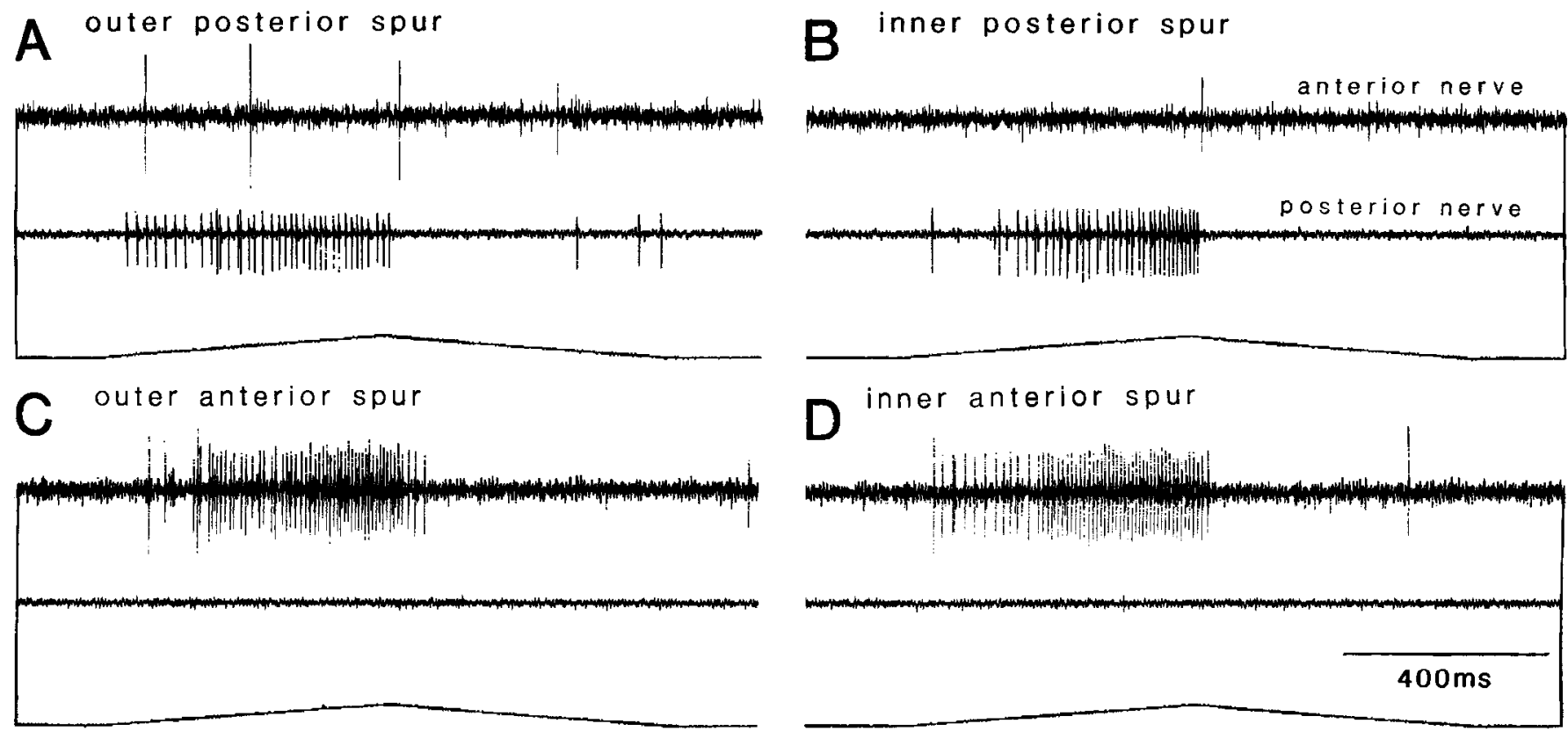

Figure 2. A single receptor cell monitors the movement of a spur. Each of the 4 spurs is displaced through an angle of $10^{\circ}$ in an imposed triangular function. An upward deflection of the lower traces in this and subsequent figures indicates that the tip of a spur is moved upwards. Afferent spikes are recorded extracellularly from the anterior (upper traces) and posterior (middle traces) tibial nerves. $A$, Outer posterior spur is moved. $B$, Inner posterior spur is moved, and the afferent spikes are now of different amplitude, indicating that they belong to a different axon. $C$, Outer anterior spur. $D$, Inner anterior spur. Nerve 5 was cut close to the metathoracic ganglion, so that all the spikes recorded belong to sensory neurons. Other spikes in the anterior nerve originate from other receptors at the tibiotarsal joint.

and stabilized on a wax-coated steel platform, and the thoracic cavity was perfused with a constant flow of saline. Microelectrodes filled with $2 \mathrm{M}$ potassium acetate were driven across the sheath of the metathoracic ganglion, which had been treated with a $1 \%(\mathrm{wt} / \mathrm{vol})$ solution of protease (Sigma Type XIV) for $2 \mathrm{~min}$, and into the somata of motor neurons and spiking local interneurons. The motor neurons were identified by correlating their intracellularly recorded spikes with efferent spikes recorded extracellularly in the tibia, or with potentials recorded extracellularly by pairs of $50 \mu \mathrm{m}$ steel wires insulated, except for their tips, in particular muscles. A depolarizing current applied through the microelectrode that induced spikes would also evoke a specific movement of the tarsus or potentials in a particular leg muscle.

A spur was moved in waveforms of controlled amplitude (maximum $20^{\circ}$ ) and frequency (maximum $10 \mathrm{~Hz}$ ) by waxing its tip, which bears no sensilla, to a small pin that was in turn attached to the arm of a vibrator (Derritron VP2 MM). Alternatively, the tip of a spur was pushed by a hand-held pin. The spikes of receptors in the spurs were recorded by 2 pairs of hook electrodes, made from $100 \mu \mathrm{m}$ silver wire, placed under nerves in the tibia. The posterior nerve, branch $b$ of nerve $5 b 2$ (see Heitler and Burrows, 1977; Pflüger, 1980) is the smallest and is purely sensory, while the anterior nerve, branch a of N5b2, also contains motor axons of muscles moving the tarsus. The conduction velocity of the afferents was measured between a pair of electrodes in the distal tibia and another on nerve 5 just before it entered the metathoracic ganglion. Recordings were stored on magnetic tape for later display on a Gould ES 1000 recorder or for photography from a storage oscilloscope.

Sensilla on the surface of a spur were revealed by scanning electron microscopy (using a Cambridge S4 Stereo-Scan) of legs that had been dehydrated and then coated with gold. Receptors inside the spurs were stained by allowing a $6 \%$ solution of hexamine cobaltic chloride to pass into their axons in nerves $5 \mathrm{~b} 2 \mathrm{a}$ and $\mathrm{b}$, cut some $3 \mathrm{~mm}$ from the proximal end of the spurs. Diffusion times of $48 \mathrm{hr}$ at $10^{\circ} \mathrm{C}$ were used, followed by development of the stain (Pitman et al., 1972) and its intensification with silver (Bacon and Altman, 1977). Thirty hindlegs from 15 locusts were stained. Recordings from interneurons and motor neurons were successfully made from 14 locusts.

\section{Results}

\section{General features of the spurs}

A conspicuous feature of the tibia of a hindleg is the 2 rows of fixed, stiff but hollow cuticular spines on the dorsal surface.
Each spine bears sensilla and is darkened at its pointed end. Near the joint with the tarsus are 4 longer and stouter movable structures that have been called spurs (Snodgrass, 1935) (Fig. 1). They are arranged more ventrally, one pair on the anterior surface, the other on the posterior surface (Fig. 1A). One spur in each pair originates more laterally and is called the outer spur, the other more medially and is called the inner spur. All 4 spurs are longer than the spines, and the 2 posterior spurs are consistently longer than the anterior ones. The 2 outer spurs have a group of trichoid sensilla on their inner face but fewer on their outer surface, whereas the reverse pertains for the inner spurs (Fig. 1C). A few shorter basiconic sensilla and a few campaniform sensilla are also present.

The 2 outer spurs subtend an angle of approximately $130^{\circ}$ to the longitudinal axis of the tibia and the 2 inner ones an angle of approximately $150^{\circ}$. None of the spurs touches the ground when the locust is standing on a flat horizontal surface, but, because of their greater length, the posterior spurs come closest to doing so. The spurs cannot be moved actively by contractions of the levator and depressor tarsi muscles, which lie nearby, but can be moved passively. All the spurs are mounted in flexible sockets that allow the 2 outer spurs to move in a proximodistal direction through approximately $70^{\circ}$ and the 2 inner ones through $50^{\circ}$ (Fig. $1 B$ ). In a dorsoventral direction, the resting position is at the ventral extreme, so that only a dorsal movement of $50^{\circ}$ is possible for the outer spurs and $30^{\circ}$ for the inner ones. For a standing locust, it is easier to describe a movement as upwards with some lateral components.

\section{Innervation of the spurs}

The 2 anterior spurs are innervated by a branch of nerve $5 b 2 a$, the anterior tibial nerve, and the 2 posterior ones by a branch of nerve $5 b 2 b$, the posterior tibial nerve. Cobalt staining reveals these nerves to innervate a campaniform sensillum at the base of each spur that has a $20 \mu \mathrm{m}$ diameter receptor cell body and a dendrite spanning the articulation to insert in the cuticle of the spur (Fig. 1, D, F, G). Also stained in a spur are single cell bodies associated with tactile hairs and campaniform sensilla. 

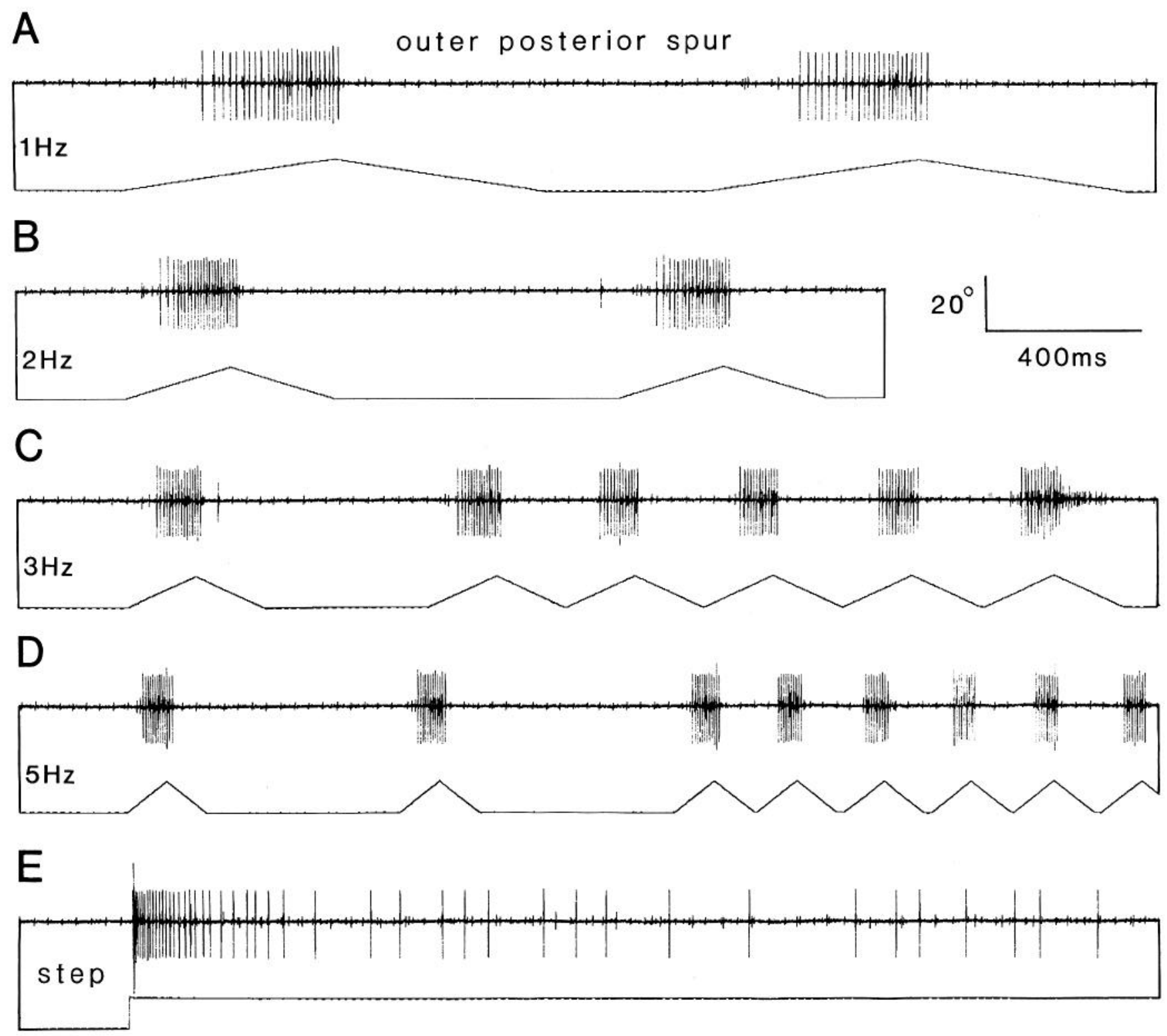

Figure 3. Response of the receptor in the outer posterior spur to upward movements at different velocities but of the same $10^{\circ}$ amplitude. $A-D$, Movements in a triangular function at $1,2,3$, and $5 \mathrm{~Hz}$, respectively. $E$. Step function to a new maintained, more proximal, position at which the afferent spikes adapt within a few seconds. At the start of this movement, a large spike from an unidentified receptor is present. During some of the triangular movements, other afferents from hairs may also be activated. Recordings were made extracellularly from the posterior tibial nerve, after nerve 5 had been cut close to the metathoracic ganglion. Small tonic spikes are from an unknown receptor.

In the scanning electron microscope an invagination of the cuticle resembling a campaniform sensillum is seen at the base of the spur that is larger than that of other nearby campaniform sensilla (Fig. $1 E$ ). In external appearance the receptor thus resembles the specialized campaniform sensillum at a femoral tactile spine in the cockroach called a socket sensillum (Chapman, 1965; French and Sanders, 1981). It is from a receptor of this type that our recordings of afferent spikes originate.

\section{Response of spur receptors}

An upward movement of the outerposterior spur evokes a burst of spikes in the posterior tibial nerve (Fig. $2 A$ ). Only one amplitude of spike is recorded throughout the movement, if hairs on the spur or at its base are not deflected, indicating that a single receptor cell has been activated. This receptor cell shows a directional sensitivity by responding briskly as the spur is moved upwards but only sporadically as it is moved downwards.
As the amplitude of movement is increased, the frequency of spikes also increases. An upward movement of the inner posterior spur also evokes a burst of spikes that differ in amplitude from those from the outer spur, indicating that they are from a different axon (Fig. $2 B$ ). When both spurs are moved together, 2 spike amplitudes can be seen, and frequencies of spikes greater than those sustainable by a single axon are recorded. In the same way, movement of either the outer or inner anterior spurs evokes bursts of spikes in the anterior tibial nerve (Fig. 2, C,D). The receptors from each of the 4 spurs thus give similar responses to a particular movement. The small differences in the frequency or number of spikes evoked probably reflect differences in the movements that were applied; the flexible mounting of a spur means that both the starting point and direction of movement are difficult to reproduce. The combined anatomical and physiological evidence therefore indicates that each spur has its own receptor to monitor its movements. 

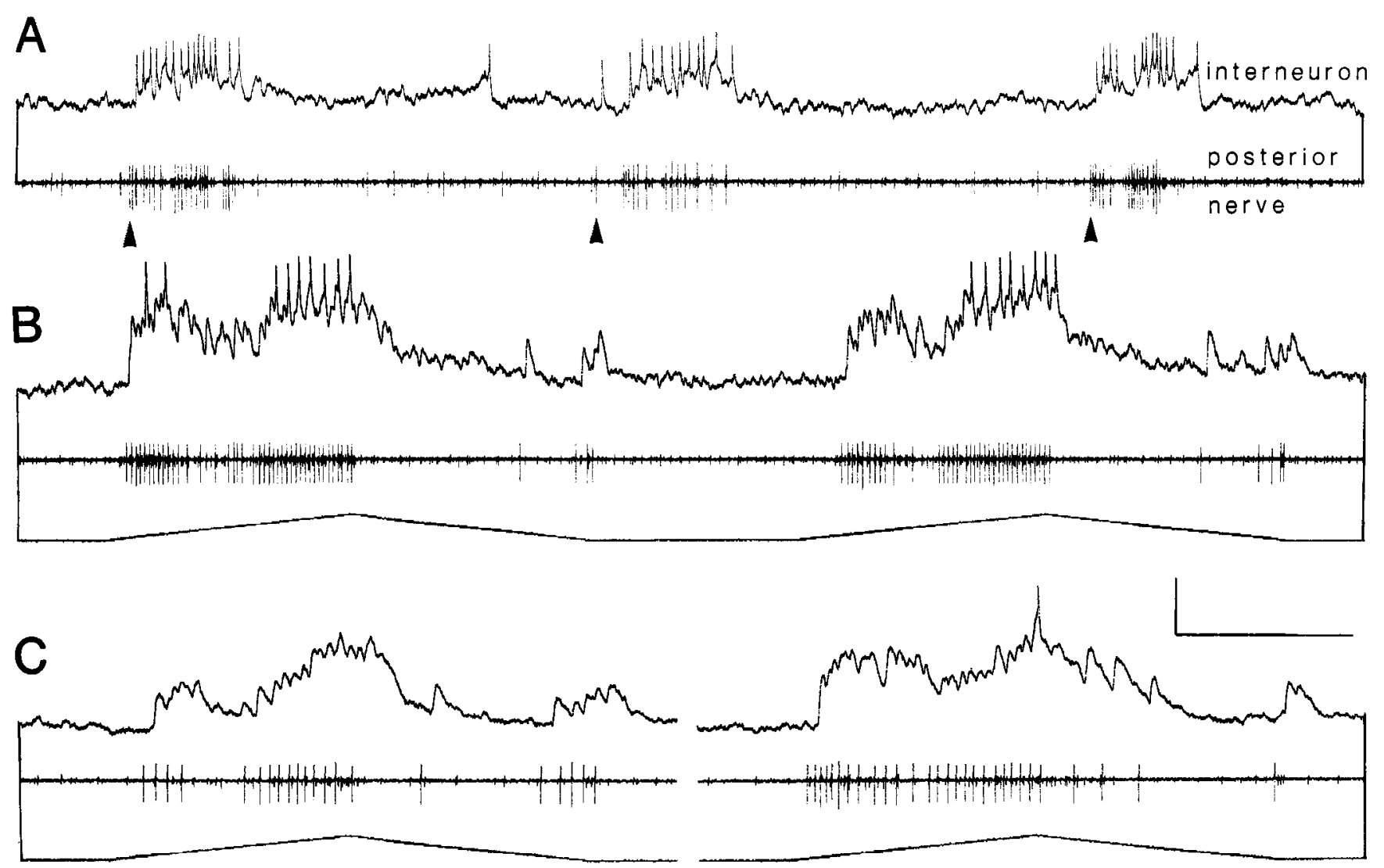

Figure 4. Movement of the outer posterior spur excites a spiking local interneuron. $A$, Each time that the spur is moved upwards (arrowheads), the afferent neuron spikes and the interneuron depolarizes and spikes. One interneuron spike occurs between the first 2 movements of the spur. $B$ and $C$, Spur is moved in controlled waveforms, which in this locust did not result in a smooth change in the frequency of the afferent spikes, while the interneuron is hyperpolarized to an unknown potential with a steady current injected through the recording electrode using a bridge circuit. $B$, Two $0.5 \mathrm{~Hz}$ movements. $C$, More hyperpolarizing current is applied and two $1 \mathrm{~Hz}$ movements are shown on an expanded time scale. All spikes from the spur receptor are followed by depolarizing potentials in the interneuron. Smaller spikes of afferents from hairs on the spur and at its base also occur during the movement and contribute to the overall depolarization. The small tonic spikes in $A$ and $B$ do not, however, evoke potentials in the interneuron. Vertical calibration: $5 \mathrm{mV}$ and $10^{\circ}$; horizontal: $A$ and $B, 800 \mathrm{msec} ; C, 400 \mathrm{msec}$.

To test how the receptors in the spurs might respond during walking, each was moved at a range of frequencies up to $5 \mathrm{~Hz}$, a value that exceeds the highest observed stepping frequency (Burns, 1973). During a $1 \mathrm{~Hz}$ movement, a maximum frequency of $80 \mathrm{~Hz}$ occurs when a spur is moved upwards (Fig. $3 A$ ). As the frequency of movement is increased, so is the frequency of spikes (Fig. 3, B, C), until at $5 \mathrm{~Hz}$ a phasic burst of spikes with frequencies of $150 \mathrm{~Hz}$ occurs at each movement (Fig. 3D). By contrast, maintained displacement of a spur, such as might occur during a change in posture, evokes a burst of spikes that adapts to one-third of its initial frequency within $200 \mathrm{msec}$ (Fig. $3 E$ ). After a further $1 \mathrm{sec}$, the spikes occur only sporadically and stop completely within 3 sec. At all likely stepping frequencies, therefore, the receptors will respond phasically and provide the CNS with information about the amplitude, velocity, and direction of movement.

\section{Afferent connections with spiking local interneurons}

Movements of a spur excite particular spiking local interneurons with cell bodies at the ventral midline of the metathoracic ganglion. One of these interneurons spikes each time that the outer posterior spur is moved (Fig. 4A). The pattern of spikes in the interneuron is a reflection of the pattern of spikes in the spur afferent; when the afferents spike at high frequency, the interneuron also spikes rapidly, and when the afferent burst is interrupted, so too is that in the interneuron (Fig. 4A).

If a steady hyperpolarizing current is applied to the interneu- ron while the spur is moved, a clear correlation emerges between the afferent spikes and depolarizing potentials in the interneuron (Fig. 4B). The potentials summate and may lead directly to a spike in the interneuron. When viewed on an expanded time scale, each afferent spike is seen to be associated with a large depolarizing potential in the interneuron (Fig. 4C). The first potential is the largest, and at this level of hyperpolarization, it can be $4 \mathrm{mV}$ in amplitude. Subsequent potentials are smaller, as they summate and depolarize the interneuron. The amplitude of a potential thus depends on the membrane potential of the interneuron. Other potentials may also contribute to the overall depolarization and are associated with the small spikes recorded in the tibial nerve. They are afferents of hairs on, or near, the spur that are displaced when the spur is moved.

A depolarizing potential that follows an afferent spike has a rise time of $8 \mathrm{msec}$ and a decay time to half-amplitude of 25 msec (Fig. 5A). In the experiment shown in Figure 5, each afferent spike is followed at a constant latency of $24 \mathrm{msec}$ by a depolarizing potential in the interneuron (Fig. $5 B$ ). The afferent spike, which is conducted at a velocity of $2.3 \mathrm{~m} / \mathrm{sec}$, is recorded approximately $52 \mathrm{~mm}$ from the entry of nerve 5 into the metathoracic ganglion. Thus, $22.6 \mathrm{msec}$ of this delay is explained by the time taken for the afferent spike to reach the ganglion. The remaining $1.4 \mathrm{msec}$ of central delay consists of the time needed for conduction of the spike a distance of 300-400 $\mu \mathrm{m}$ (approximately $0.2 \mathrm{msec}$ ) to the synaptic sites, and the synaptic delay. The short time available for synaptic transmission suggests that 


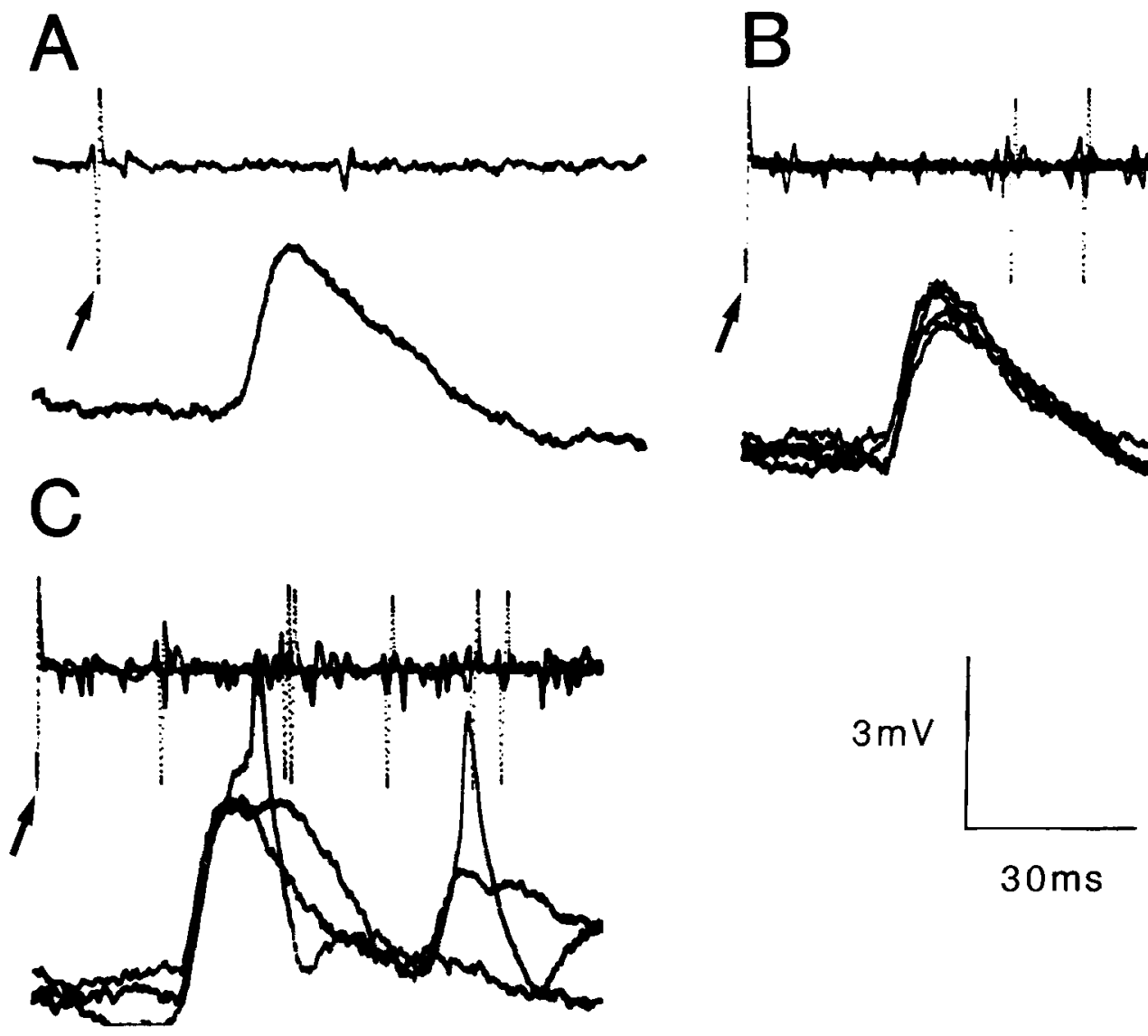

Figure 5. Spikes of the afferent from the receptor in the outer posterior spur evoke depolarizing synaptic potentials in the spiking local interneuron shown in Figure 4. The afferent spikes (the first one in each upper trace is marked with an arrow) are recorded extracellularly from the posterior tibial nerve $52 \mathrm{~mm}$ from the metathoracic ganglion. The interneuron is held hyperpolarized by a steady current to suppress most of its spikes. $A$, Single afferent spike evokes a large depolarizing potential in the interneuron. $B$, Superimposed sweeps triggered by the afferent spikes show the consistent occurrence and latency of the depolarizing potential. $C$, Superimposed sweeps in which 2 of the potentials evoke spikes in the interneuron. The interneuron is impaled in its soma, which is distant from the spike initiating and synaptic input sites.

the afferent from this spur receptor synapses directly upon the interneuron.

The summating depolarizing potentials can evoke a spike in the interneuron (Fig. $5 \mathrm{C}$ ). The membrane potential at which the spike arises can differ, indicating that the recording site in the soma is distant from the spike-initiating sitc. The ability of the potential to evoke a spike and the dependence of its amplitude upon the membrane potential (Fig. 4) indicate that it is a chemically mediated EPSP.

The 4 spurs differ dramatically in the ability of their receptors to excite this interneuron (Fig. 6). Movement of the 2 anterior spurs has no effect (Fig. $6, A, B$ ). The inner posterior spur produces a depolarization of the interneuron but fails to evoke any spikes (Fig. 6C). The outer posterior spur is the most effective, producing depolarizations that are twice the amplitude of those evokcd by the inner posterior spur and evoking spikes (Fig. $6 D$ ). The individual EPSPs associated with the afferent spikes of the 2 posterior receptors are of different amplitudes, even though both occur with the same latency (Fig. 6, E, F). The EPSP from the outer posterior spur is $3-4 \mathrm{mV}$ as recorded in the cell body, whereas that from the inner posterior spur is only $1 \mathrm{mV}$. While some of this difference may simply be a reflection of the spatial arrangement of the synapses of the 2 afferents on the interneuron relative to the recording site, the greater efficacy of the larger EPSP from the outer spur is emphasized by its ability to evoke spikes (Fig. 6D).

This interneuron is also excited by hairs on the distal posterior surface of the tibia, while hairs on the anterior surface have no effect. Its receptive field is thus organized about the anteroposterior long axis of the leg. Recordings from a spiking local interneuron with these properties have been made in 3 locusts. Possibly all recordings are from the same interneuron or, alternatively, they arc from a small number of interneurons with similar properties.

\section{Different spurs excite different interneurons}

The 2 anterior spurs that have no effect on the preceding interneuron excite different spiking local interneurons, which are in turn unaffected by movements of the posterior spurs. The receptive fields of the 2 groups of interneurons are thus complementary. Moving the outer anterior spur evokes bursts of spikes in its receptor that are always accompanied by bursts of spikes in an interneuron (Fig. 7, $\boldsymbol{A}, \boldsymbol{B}$ ). Each movement is also accompanied by inhibition of the single levator tarsi motor neuron whose axon runs in the anterior tibial nerve and generates the large spike in the recording (Fig. 7). When the interneuron is hyperpolarized, a clear correlation becomes apparent between the afferent spikes and depolarizing potentials (Fig. 7C). Each afferent spike is followed by a large depolarizing potential in the interneuron at a constant latency (Figs. $7 D ; 8, A, B$ ). The afferent spike is conducted at a velocity of $2.1 \mathrm{~m} / \mathrm{sec}$, so that all but 2.2 msec of the latency is explained by the time taken for the spike to travel from the recording electrode to the metathoracic ganglion. The short and consistent central latency suggests that this 

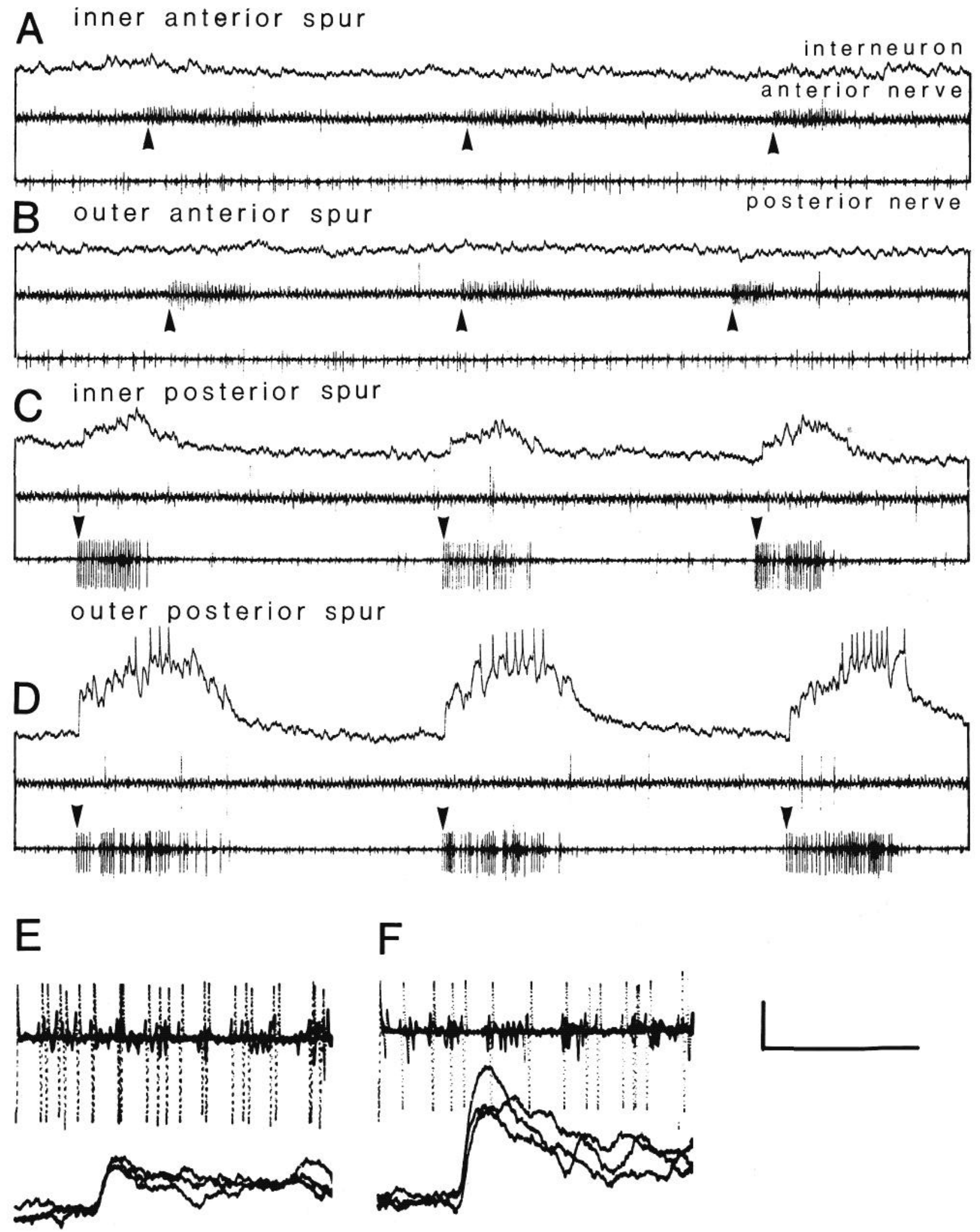

Figure 6. Receptors in different spurs vary in their effectiveness in exciting the spiking local interneuron shown in Figures 4 and 5 . Neither the inner $(A)$ nor the outer $(B)$ anterior spurs evoke a depolarization in this interneuron when they are moved upwards (arrows). Both the posterior spurs do, however, evoke a depolarization. $C$, Inner posterior spur evokes a depolarization but no spikes. $D$, Outer posterior spur is the most effective and evokes a large depolarization and spikes. Extracellular recordings are from the anterior (second traces) and posterior (third traces) tibial nerves. $E$, Superimposed sweeps triggered by the spike of the inner posterior spur (upper trace) reveal an EPSP at constant latency. $F$, Sweeps triggered by the spike of the outer posterior spur reveal an EPSP of much greater magnitude but with the same latency. Vertical calibration: $A-D$, $5 \mathrm{mV} ; E$ and $F, 1.5 \mathrm{mV}$; horizontal: $A-D, 800 \mathrm{msec} ; E$ and $F, 50 \mathrm{msec}$. 

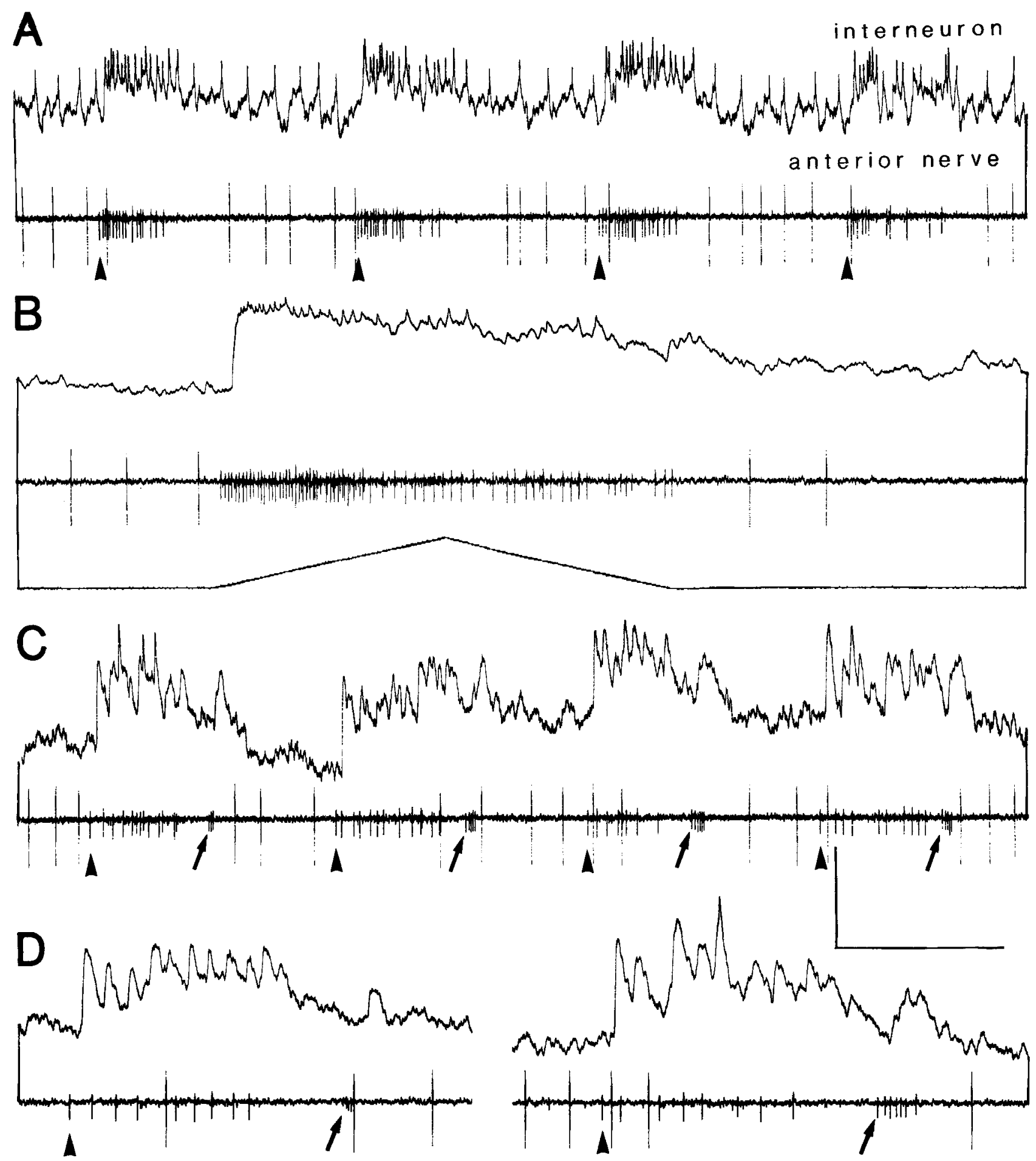

Figure 7. Movement of the outer anterior spur excites a different spiking local interneuron. A, Upward movements of this spur (arrowheads) evoke bursts of sensory spikes in the anterior tibial nerve (lower trace), increase the frequency of spikes in the interneuron, and inhibit the spikes of the levator tarsi motor neuron (large spikes). B. Interneuron is hyperpolarized and the spur moved in a $1 \mathrm{~Hz}$ waveform (lower trace), which leads to a rapid depolarization and spikes in the interneuron. $C$. Interneuron is hyperpolarized further to reveal the depolarizing potentials that occur whenever the sensory neuron spikes. $D$. Two movements of the spur on an expanded time scale. Each sensory spike from the spur receptor is followed by an EPSP in the interneuron. In $C$ and $D$ spikes from hairs at the base of the spurs (diagonal arrows) also depolarize the interneuron. Recording in $B$ was made late in the experiment when the recording had slightly deteriorated. Vertical calibration: $5 \mathrm{mV}$ and $20^{\circ}$; horizontal: $A$ and $C, 800 \mathrm{msec} ; B$ and $D, 400 \mathrm{msec}$.

afferent connects directly with this interneuron. The evoked depolarizing potential may sometimes lead to a spike in the interneuron (Fig. 8C), and its amplitude is enhanced when the interneuron is hyperpolarized. It is thus a chemically mediated EPSP.
Other afferents on the anterior surface of the tibia may also excite this interneuron when a spur is moved. For example, a burst of small spikes, which probably originates from hairs at the base of the spur, is followed by a depolarization of the interneuron (Fig. 7, $C, D$ ). Triggering a signal averager from the 

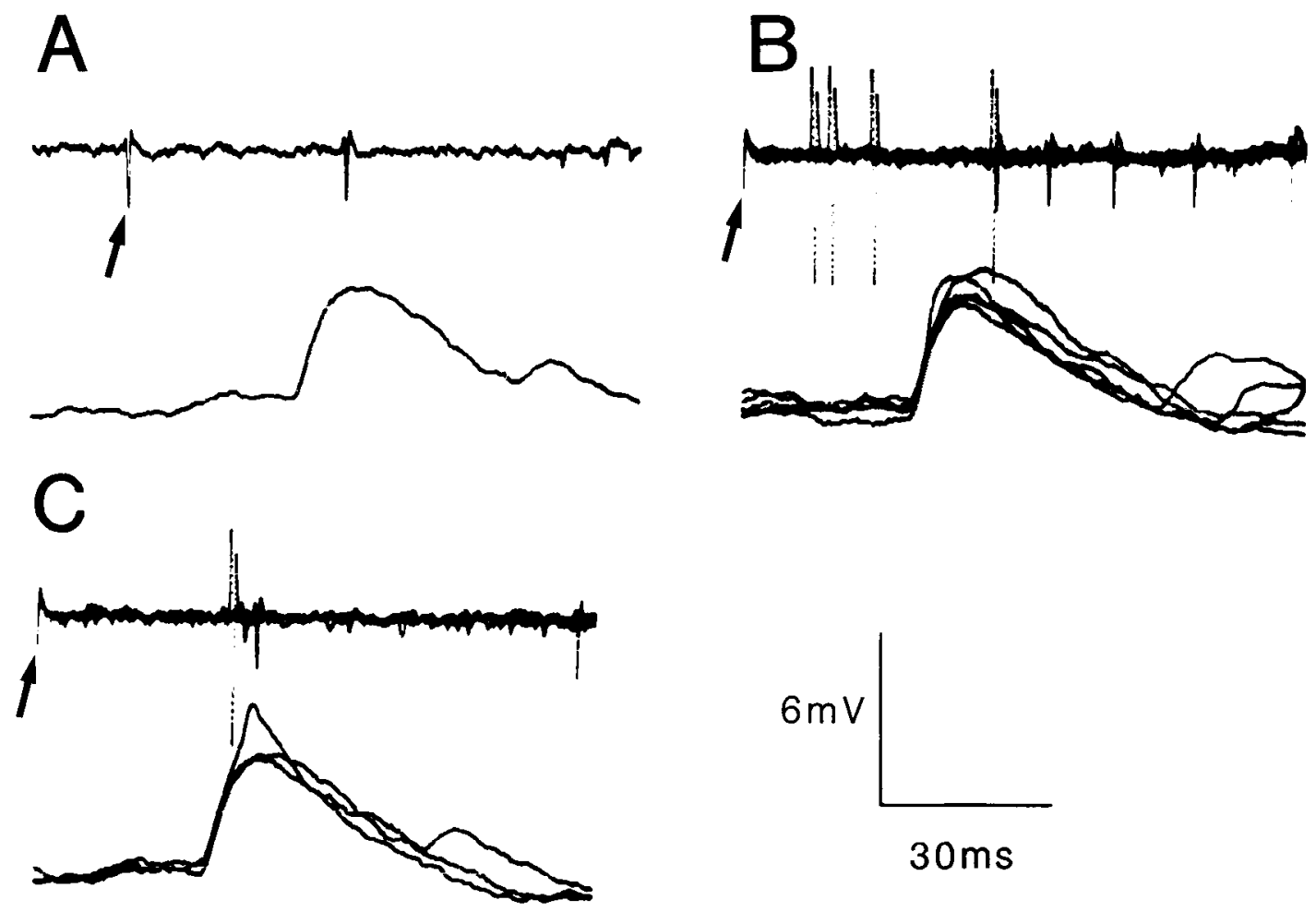

Figure 8. Spikes of the afferent from the receptor in the outer anterior spur evoke depolarizing synaptic potentials in a spiking local interneuron. Afferent spikes (the first one in each upper trace is marked with an arrow) are recorded extracellularly from the anterior tibial nerve $52 \mathrm{~mm}$ from the metathoracic ganglion. The large spikes are those of the levator tarsi motor neuron. The interneuron is held hyperpolarized by a steady current. $A$, Single afferent spike is followed by an EPSP (lower trace). B, Superimposed sweeps, triggered by pulses from a window circuit through which the nerve recording is passed, show the consistent occurrence and latency of the EPSP. $C$. Superimposed sweeps in which an EPSP gives rise to a spike in the interneuron.

spikes of an individual hair shows that they evoke an EPSP in the interneuron with a latency some $3 \mathrm{msec}$ longer and an amplitude one-third of that evoked by the spur receptor. The longer latency is explained by the slower conduction velocity of these afferents, so that the evidence again indicates that the connection is direct.

\section{Reflex effects upon leg motor neurons}

An upward movement of any of the 4 spurs evokes a consistent reflex inhibition of the single motor neuron that innervates the levator tarsi muscle (Fig. 9). For example, movements of the inner posterior spur interrupt the tonic spikes in the motor neuron (Fig. 9A). The spikes of the receptor afferent are associated with large hyperpolarizing IPSPs that when summed can increase the membrane potential of the motor neuron by some 8-9 $\mathrm{mV}$. Unlike any of the spiking local interneurons, the levator motor neuron is affected by both posterior spurs and by both anterior ones. Thus, movement of the outer posterior spur (Fig. 9B), as well as the inner (Fig. 9C) or outer (Fig. 9D) anterior spurs, also evokes a reliable inhibition that can follow at frequencies of $5 \mathrm{~Hz}$. The efficacy of the 4 spurs in inhibiting the motor neuron is similar in all animals, and occasions when 1 spur was less effective than the others can be attributed to the different behavioral contexts in which the movement was imposed. For example, the tarsus might be held fully levated so that dorsal tarsal and ventral tibial receptors were excited, or the animal might be attempting other movements.

A sequence of spikes in a spur afferent is followed by a group of IPSPs in the levator tarsi motor neuron (Fig. 10A), but there is no strict 1:1 correlation between an individual afferent spike and an IPSP (Fig. 10B). When a few sweeps of the oscilloscope are triggered by the afferent spikes and superimposed, no IPSP consistently follows each spike (Fig. 10C). Superimposing many sweeps, however, shows that an IPSP tends to follow an afferent spike with a central latency of approximately $3 \mathrm{msec}$ (Fig. 10D). Measurements of the latency to the EPSP in a spiking local interneuron and to the IPSP in the levator motor neuron have, however, not been made in the same locust. When afferent spikes trigger a signal averager, an IPSP of variable latency and amplitude is revealed. This is in contrast to the EPSP of consistent latency and amplitude that is revealed by similar methods in a spiking local interneuron. These results suggest that the afferents do not connect directly with the motor neuron, but instead affect it through a pathway that need involve no more than 1 interposed spiking local interneuron. Hyperpolarizing a spiking local interneuron that receives inputs from spur afferents to suppress its spikes reduces the inhibition of the levator motor neuron when the appropriate spur is moved. The reflex is not completely suppressed, implying that several interneurons contribute to the inhibition.

Motor neurons innervating other tarsal muscles are unaffected by movements of the spurs. For example, movements of the inner posterior (Fig. 11A) or outer anterior spur (Fig. 11B) have no effect on a slow depressor tarsi motor neuron. Similarly, movements of the inner (Fig. $11 C$ ) or outer (Fig. $11 D$ ) posterior spurs do not alter the frequency of spikes in a slow motor neuron to the retractor unguis muscle.

Motor neurons that innervate muscles moving more proximal joints of the leg may be excited by movement of the spurs. One of the flexor tibiae motor neurons receives a sequence of depolarizing potentials that do not usually lead to spikes and are not linked in a 1:1 fashion to the afferent spikes from the outer or inner posterior spurs (Fig. 11, E, F). The slow and fast motor neurons to the extensor tibiae muscle are, however, unaffected. 

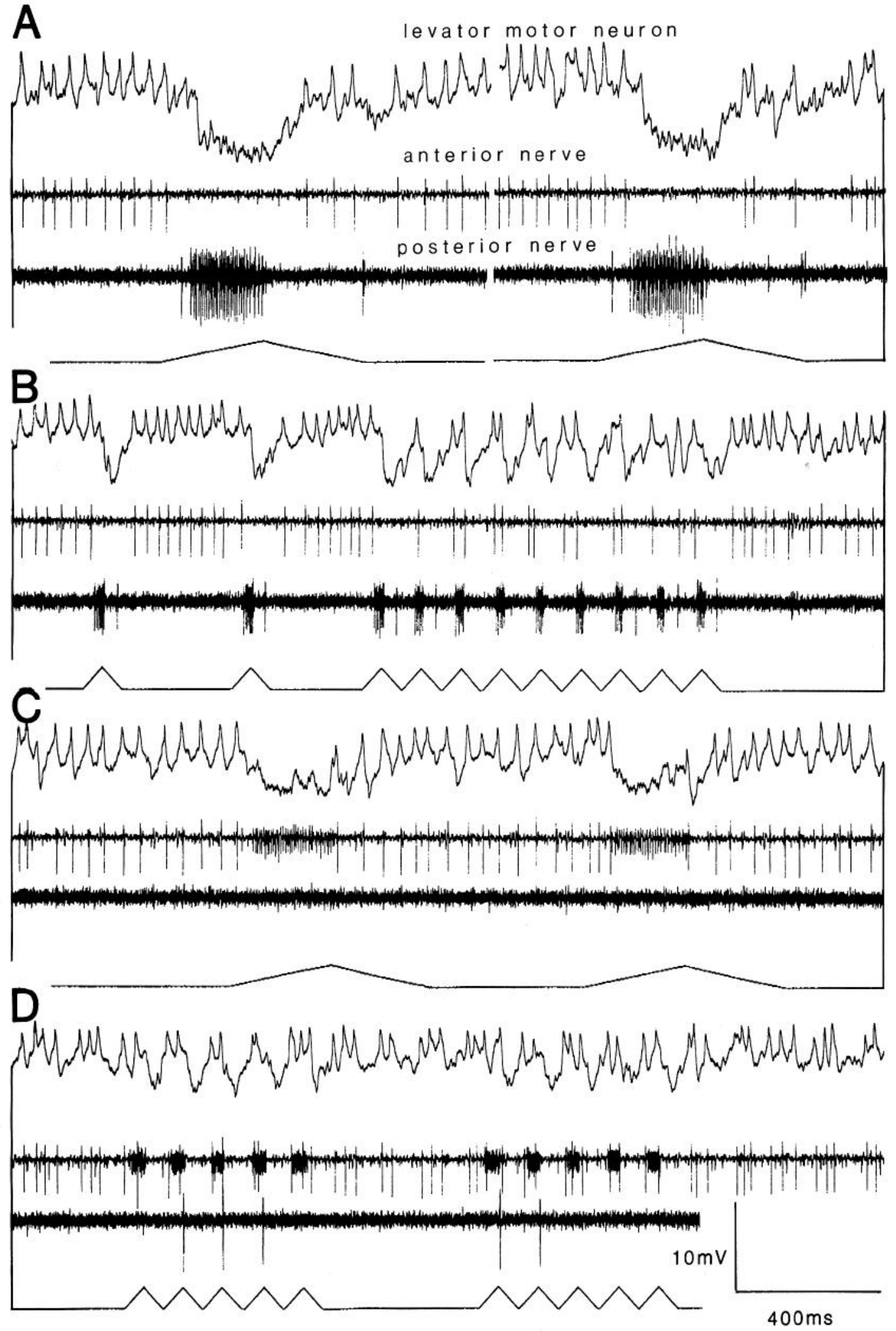

Figure 9. Spur receptors evoke a consistent inhibitory reflex effect on the single levator tarsi motor neuron. Recordings are made intracellularly from the cell body of the motor neuron (upper traces) and extracellularly from the afferents in the anterior (second traces) and posterior (third traces) tibial nerves. Anterior nerve also records the spikes of the motor neuron. $A$, Each time that the inner posterior spur is moved upwards (fourth traces) the motor neuron is inhibited. B. Moving the outer posterior spur at $5 \mathrm{~Hz}$ gives a consistent inhibition, with the motor neuron recovering to spike on each cycle. $C$, Inner anterior spur also evokes inhibition. $D$, Outer anterior spur is moved repetitively at $5 \mathrm{~Hz}$, evoking a consistent, though weaker, inhibition. 


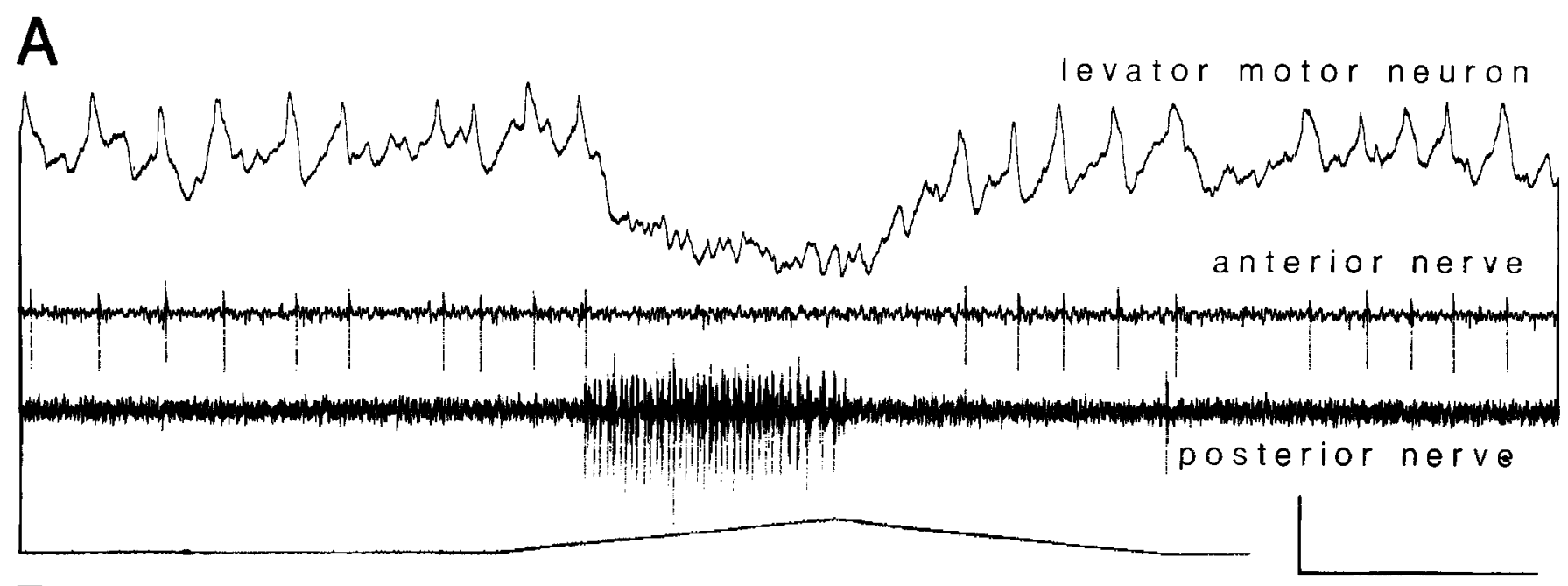

B

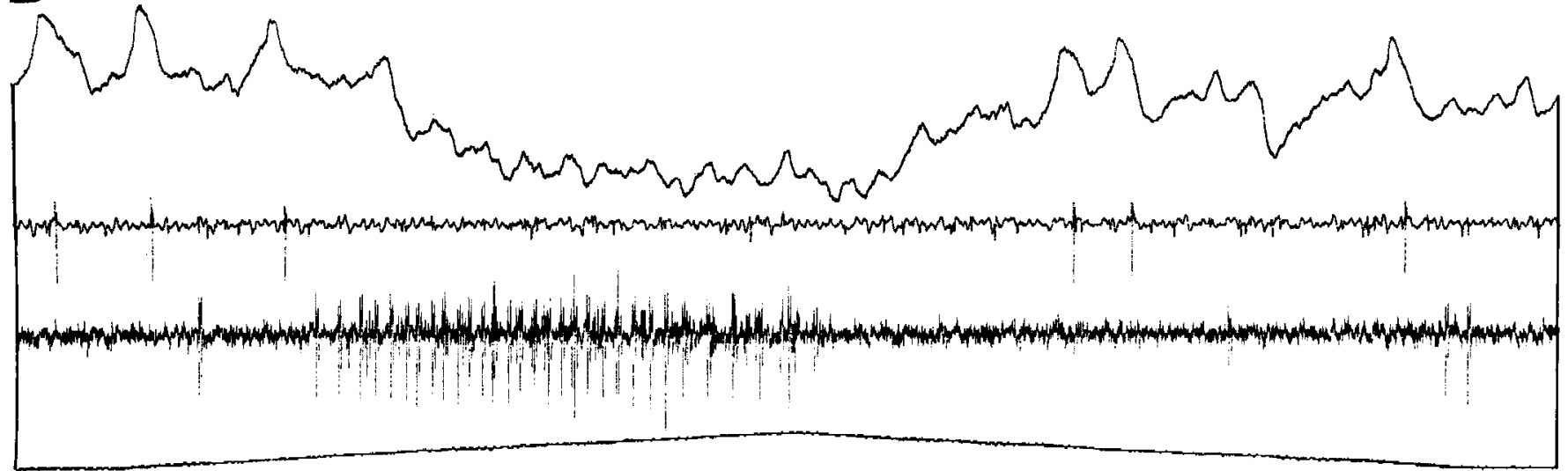

C
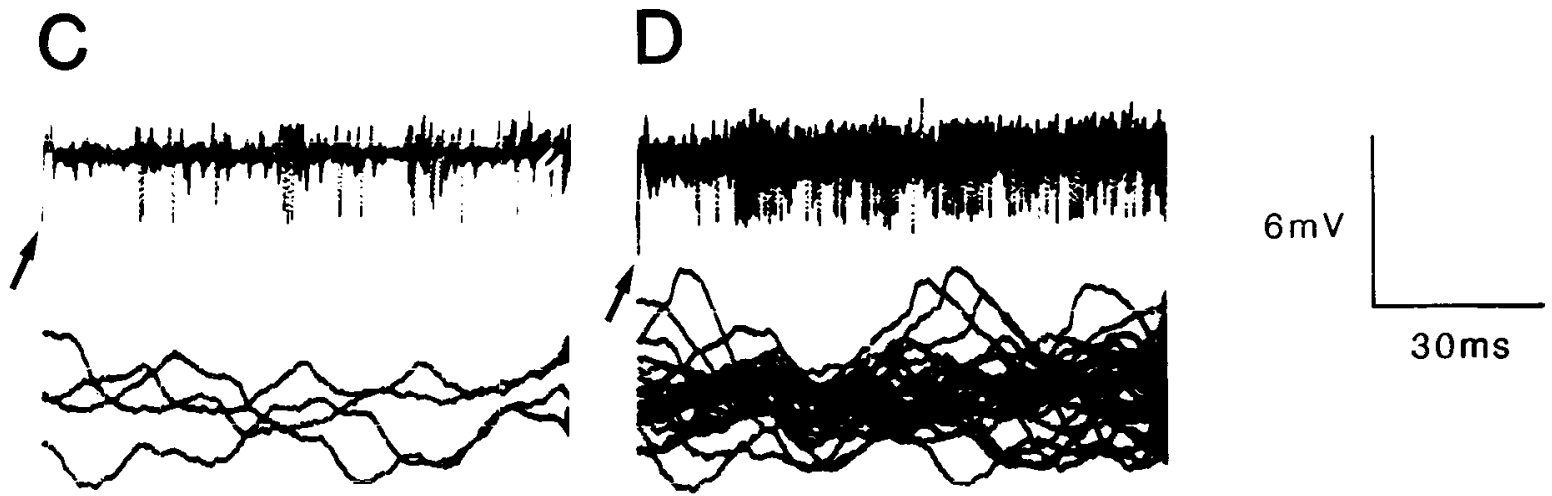

Figure 10. Inhibition in the levator tarsi motor neuron is not a direct effect of the afferent input. A, Movement of the inner posterior spur excites the receptor afferent and inhibits the motor neuron. $B$, Same movement on a faster time scale shows no strict correlation between afferent spikes and IPSPs in the motor neuron. $C$, Four sweeps of the oscilloscope triggered by the afferent spikes reveal no consistent IPSP. $D$, Many superimposed sweeps, however, show a tendency for an IPSP to occur with a particular latency. Vertical calibration: $A$ and $B, 10 \mathrm{mV}$; horizontal: $A, 400 \mathrm{msec}$; $B, 200$ msec.

The most reliable excitatory effect was found in a motor neuron suspected on the basis of myogram recordings to be an anterior rotator of the coxa (Fig. $11, G, H$ ). This motor neuron is depolarized and spikes as a result of summed EPSPs, which are again not linked in a 1:1 fashion with the afferent spikes.

\section{Discussion}

The spiking local interneurons are known to be the primary integrators of signals from hairs (trichoid sensilla) and other campaniform sensilla on a leg of a locust (Siegler and Burrows, 

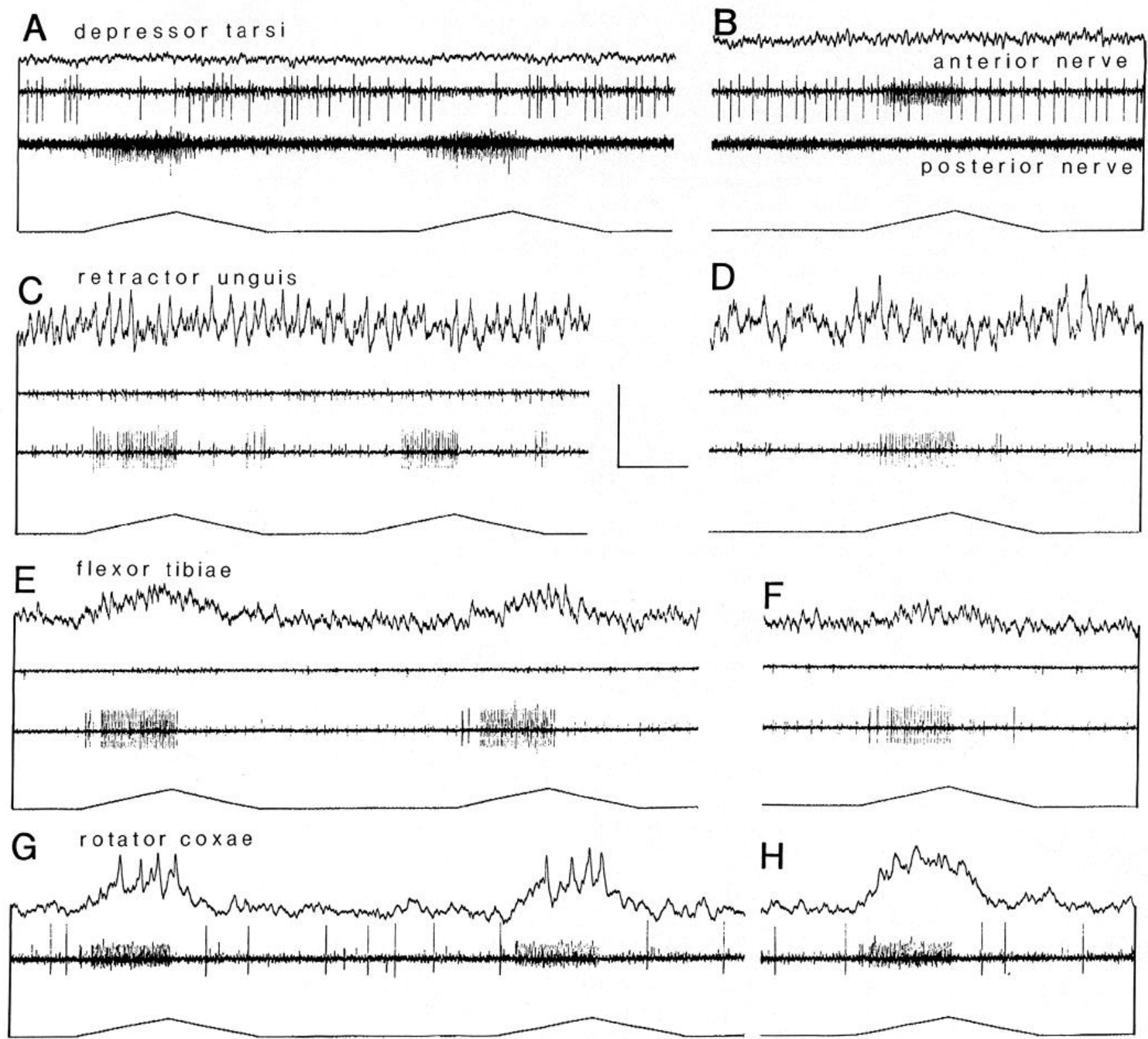

Figure 11. Reflex effects of spur afferents on other leg motor neurons. A depressor tarsi motor neuron is not affected by movement of the inner posterior $(A)$ or outer anterior spur $(B)$. Similarly, a retractor unguis motor neuron is not affected by a movement of the inner $(C)$ or outer posterior spur $(D)$. A flexor tibiae motor neuron is depolarized when the outer $(E)$ or inner $(F)$ posterior spur is moved. $G$, An unidentified motor neuron of a coxal muscle is depolarized and spikes when the outer anterior spur is moved. $H$, Synaptic potentials contributing to the depolarization are revealed when this motor neuron is hyperpolarized. Vertical calibration: $10 \mathrm{mV}(A, B, G, H) ; 5 \mathrm{mV}(C-F)$; horizontal: $400 \mathrm{msec}$.

1983). Here, they are shown to integrate information from the movable spurs at the tibiotarsal joint. A spur is unusual in that it is moved only by contact with external objects and not by muscular action. It is not simply a grossly enlarged hair, because there are other sensilla on its surface. The receptor at its base is sensitive to the direction, velocity, and amplitude of movement, being excited when a spur is moved upwards. It will thus signal an unusual placement of the tarsus and, because its response is phasic, it should produce a burst of spikes on each step.

\section{Connections of the afferents}

The effect on spiking local interneurons by moving a spur is either to raise the frequency of tonic spiking or to induce a burst of spikes if the cell has previously been silent. Each afferent spike is consistently followed by an EPSP whose amplitude depends upon the membrane potential of the interneuron and that can itself lead directly to a spike. The latency with which an EPSP follows a spike is always consistent, and the estimated delay within the metathoracic ganglion is between 1.4 and 2.2 msec for different interneurons in different locusts. These observations suggest that the afferents synapse directly upon the interneuron, but this interpretation invites caution. Typically, the spikes were recorded $52 \mathrm{~mm}$ from the ganglion, but this distance can be measured with an accuracy of only $\pm 20 \%$. It is likely that the length of the nerve, and hence also the velocity, is always underestimated. The nerve is elastic and longer than the overlying exoskeleton, which is actually measured, to allow freedom of movement at the femorotibial joint. Over this distance, the spikes of the spur afferents are conducted at a velocity 
of $2.1-2.3 \mathrm{~m} / \mathrm{sec}$. Most of the measured latency thus consists of the time taken for the spike to reach the ganglion, so that small errors here will lead to overestimates in the central delay. It is to these causes that we attribute the differences in estimated central latencies for the 2 interneurons described, and not to different pathways within the ganglion.

Once at the ganglion, the spike must then travel to synaptic sites that are probably in the ventral association area. It is in this region of neuropil that the spiking local interneurons have a prominent projection (Siegler and Burrows, 1984) and where their branches have a preponderance of input synapses (Watson and Burrows, 1985). Afferents from hairs and campaniform sensilla on the leg also project to this region (Pflüger et al., 1981). Thus, the time remaining is probably sufficient for only 1 synapse to be involved. In the same ganglion, synaptic delays comparable to this are found between hair afferents and local interneurons (Siegler and Burrows, 1983) and between a wing stretch receptor and flight motor neurons (Burrows, 1975). Somewhat shorter delays of $0.9 \mathrm{msec}$ have been found for the connection between spiking local interneurons and motor neurons (Burrows and Siegler, 1982), but then pre- and postsynaptic recording sites were close to each other.

The experiments do not imply, however, that these are the only connections made by the afferents from the spurs. Other pathways may also exist. For example, afferents from a hair plate on a cockroach leg, excited when the trochanter is levated, project directly to leg motor neurons (Pearson et al., 1976), and the single afferent from a stretch receptor at the base of a locust wing, excited when the wing is elevated, projects directly to motor neurons (Burrows, 1975) and to intersegmental interneurons (Pearson et al., 1983).

\section{Integration of the sensory signals}

The input from the spurs is segregated, so that a spiking local interneuron excited by posterior spurs is unaffected by anterior ones and vice versa. Moreover, under the same conditions when an interneuron is hyperpolarized, the input from an outer spur may evoke spikes while the input from an inner spur, despite evoking consistent EPSPs, fails to do so. Therefore, receptors within the receptive field of an interneuron are not given equal weighting. For the spur input, this unequal weighting makes functional sense. The inner spurs will be the first to be stimulated when the tarsus is placed on the ground and will supply the interneuron with a signal that must sum with other inputs to evoke spikes. A reflex adjustment of the tarsus is thus not obligatory. The outer spurs, by contrast, will only be moved by a greater misplacement of the tarsus or if the ground is particularly rough. They therefore act as high-threshold receptors and, commensurate with this, their input is given stronger weighting so that a reflex adjustment of the tarsus will follow.

The segregation of inputs from the anterior and posterior spurs and the preservation of spatial information would seem to indicate more subtle reflex effects than have so far been observed. The spurs are only a part of the whole receptive fields of the spiking local interneurons that they excite. For example, an interneuron that is excited by an anterior spur is also excited by hairs on the anterior surface of the distal tibia. Touching these hairs leads to a local postural reflex involving an anterior rotation of the coxa (Siegler and Burrows, 1986). Moving an anterior spur also leads to excitation of a motor neuron thought to innervate an anterior coxal rotator muscle.

\section{The reflex pathway}

A movement of any of the spurs that excites their afferents can lead to a reliable reflex inhibition of the single levator tarsi motor neuron. No interneurons have been found that are excited by all 4 spurs, so that those excited by the anterior spurs and those excited by the posterior spurs must converge on the motor neu- ron. To explain the pattern of IPSPs in the motor neuron when a spur is moved requires the participation of no more than 2 spiking local interneurons. This accords well with our estimates on the basis of sampling many spiking local interneurons that only a small number receive inputs from the spurs. The following evidence suggests that the reflex involves 3 neuron pathways involving a sensory neuron, a spiking local interneuron, and a motor neuron. First, the afferents would seem, according to physiological criteria, to make direct connections with a small group of spiking local interneurons. They do not synapse directly upon the levator motor neuron. Second, there is a correlation between the occurrence of afferent spikes, interneuron spikes, and inhibitory potentials in the motor neuron. Hyperpolarizing an interneuron to suppress its spikes can reduce, but does not completely abolish, the inhibition (Fig. 7), indicating again that convergence onto the motor neuron must occur. Third, some interneurons with the same receptive fields as those described here, and thus perhaps the same neurons, evoke IPSPs directly on the levator tarsi motor neuron (Burrows and Siegler, 1982, 1984). The correlation observed here between afferent spikes and IPSPs in the motor neuron, as revealed by averaging methods, is explained if a spiking local interneuron is the only element interposed between the two. Thus, an afferent spike will evoke an EPSP in an interneuron, but not every EPSP will lead to a spike. Each spike that does occur in the interneuron will, however, lead to an IPSP in the motor neuron.

The pathways to motor neurons that are excited when a spur is moved are probably more complex. The only effects on motor neurons exerted by the spiking local interneurons have so far proved to be inhibitory (Burrows and Siegler, 1982, 1984). More likely candidates to excite the motor neurons are the nonspiking local interneurons, which synapse on sets of motor neurons in patterns appropriate to achieve coordinated movements of a leg (Burrows, 1980). The pathway to the motor neurons that are excited in this reflex would therefore be expected to involve at least 4 neurons, one of them a nonspiking local interneuron.

\section{Role of the reflex}

The function of the reflex inhibition of the levator tarsi motor neuron becomes apparent in the following contexts, if the effects observed in our experiments when the leg is not supporting the weight of the body also occur when it does. First, if the locust leans to the left while walking or standing still, the anterior spurs on the left hindleg will be moved upwards and thus excited. The unequal transference of the body weight to the left hindleg must be resisted by the tibiotarsal joints among others. Second, if during walking the locust encounters rough ground that deflects a spur, the requirement is again for a firmer foothold to prevent stumbling. In both examples, the pressure exerted on the proximal part of the tarsus (the heel) will be too high and must be relieved by a depression of tarsus. This is achieved by inhibition of the levator muscle to allow an unopposed depression of the tarsus. This will raise the body and reduce the stimulation on the spurs.

Is the reflex fast enough to allow adjustment of the movement during a single step cycle? A locust walks at a frequency of between 2 and $8 \mathrm{~Hz}$ on a flat horizontal surface (Burns, 1973), but when climbing a vertical surface the frequency of leg movements falls to about $1.5 \mathrm{~Hz}$ (Pflüger, unpublished observations). The stance phase, when the ventral surface of the tarsus is in contact with the ground will thus range in duration from 100 to $400 \mathrm{msec}$. The spike of the levator tarsi motor neuron takes approximately $15 \mathrm{msec}$ to travel at a velocity of $3.7 \mathrm{~m} / \mathrm{sec}$ from the metathoracic ganglion to its muscle. The spike of the afferent from the receptor in the spur takes approximately $25-26 \mathrm{msec}$ to travel from the spur to the metathoracic ganglion. Thus, conduction of spikes in the periphery takes at least $40 \mathrm{msec}$. Within the CNS, time must be allowed for conduction to syn- 
aptic sites and for delays at 2 synapses: first at the synapse of the afferent with the spiking local interneuron and, second, at the synapse between this interneuron and the levator tarsi motor neuron. Where excitation of other motor neurons is involved, an extra synaptic delay must be added. Thus, for the spur receptors to alter the contraction of a tarsal muscle requires a reflex pathway time of some $45 \mathrm{msec}$. The inhibitory reflex pathway thus allows adjustments of the leg to be made throughout most of the present stance phase.

\section{References}

Bacon, J. P., and J. S. Altman (1977) A silver intensification method for cobalt-filled neurones in wholemount preparations. Brain Res. 138: 359-363.

Bässler, U. (1983) Neural Basis of Elementary Behavior in Stick Insects, Springer-Verlag, Berlin.

Burns, M. D. (1973) The control of walking in orthoptera. I. Leg movements in normal walking. J. Exp. Biol. 58: 45-58.

Burrows, M. (1975) Monosynaptic connexions between wing stretch receptors and flight motoneurones of the locust. J. Exp. Biol. 62: 189219.

Burrows, M. (1980) The control of sets of motoneurones by local interneurones in the locust. J. Physiol. (Lond.) 298: 213-233.

Burrows, M., and M. V. S. Siegler (1982) Spiking local interneurons mediate local reflexes. Science 217: 650-652.

Burrows, M., and M. V. S. Siegler (1984) The morphological diversity and receptive fields of spiking local interneurones in the locust metathoracic ganglion. J. Comp. Ncurol. 224: 483-508.

Burrows, M., and M. V. S. Siegler (1985) Organization of receptive fields of spiking local interneurons in the locust with inputs from hair afferents. J. Neurophysiol. 53: 1147-1157.

Burrows, M., and B. L. Watkins (1986) Spiking local interneurones in the mesothoracic ganglion of the locust: Homologies with metathoracic interneurones. J. Comp. Neurol. 245: 29-40.

Chapman, K. M. (1965) Campaniform sensilla on the tactile spines of the legs of the cockroach. J. Exp. Biol. 42: 191-203.

Cruse, H., and H. J. Pflüger (1981) Is the position of the femur-tibia joint under feedback control in the walking stick insect? II. Electrophysiological recordings. J. Exp. Biol. 92: 97-107.
Forssberg, H. (1979) Stumbling corrective reaction: A phase-dependent compensatory reaction during locomotion. J. Neurophysiol. 42: 936-953.

French, A. S., and E. J. Sanders (1981) The mechanosensory apparatus of the femoral tactile spine of the cockroach, Periplaneta americana. Cell Tissue Res. 219: 53-68.

Heitler, W. J., and M. Burrows (1977) The locust jump. I. The motor programme. J. Exp. Biol. 66: 203-219.

Pearson, K. G., D. N. Reye, and R. M. Robertson (1983) Phasedependent influences of wing stretch receptors on flight rhythm in the locust. J. Neurophysiol. 49: 1168-1181.

Pearson, K. G., R. K. S. Wong, and C. R. Fourtner (1976) Connexions between hair-plate afferents and motoneurones in the cockroach leg. J. Exp. Biol. 64: 251-266.

Pflïger, H. J. (1980) The function of hair sensilla on the locust's leg: The role of tibial hairs. J. Exp. Biol. 87: 163-175.

Pflüger, H. J., P. Bräunig, and R. Hustert (1981) Distribution and specific central projections of mechanoreceptors in the thorax and proximal leg joints of locusts. II. The external mechanoreceptors: Hair plates and tactile hairs. Cell Tissue Res. 216: 79-96.

Pitman, R. M., C. D. Tweedle, and M. J. Cohen (1972) Branching of central neurons: Intracellular cobalt injection for light and electron microscopy. Science 176: 412-414.

Siegler, M. V. S., and M. Burrows (1983) Spiking local interneurons as primary integrators of mechanosensory information in the locust. J. Neurophysiol. 50: 1281-1295.

Siegler, M. V. S., and M. Burrows (1984) The morphology of two groups of spiking local interneurones in the metathoracic ganglion of the locust. J. Comp. Neurol. 224: 463-482.

Siegler, M. V. S., and M. Burrows (1986) Receptive fields of motor neurons underlying local tactile reflexes in the locust. J. Neurosci. 6: 507-513.

Snodgrass, R. E. (1935) Principles of Insect Morphology, McGrawHill, New York.

Watson, A. H. D., and M. Burrows (1985) The distribution of synapses on the two fields of neurites of spiking local interneurones in the locust. J. Comp. Neurol. 240: 219-232. 